\title{
Road Networks and their Incomplete Representation by Network Data Models
}

\author{
Simon Scheider ${ }^{1}$, Werner Kuhn ${ }^{2}$ \\ ${ }^{1}$ Fraunhofer Institut für Intelligente Analyse und Informationssysteme (IAIS), Schloss \\ Birlinghoven, 53754 Sankt Augustin, Germany \\ simon.scheider@iais.fraunhofer.com \\ ${ }^{2}$ Institute for Geoinformatics (ifgi), University of Münster, \\ Weselerstr. 253, 48151 Münster, Germany \\ kuhn@uni-muenster.de
}

\begin{abstract}
Road networks, roads, and junctions are examples of natural language terms whose semantics can be described by affordances of their physical referents. In order to define affordances in such a way that they can be used for classifying and describing instances in a geographic database, one has to deal with the problems of informational incompleteness and limited definability. In this paper, we propose an affordance-based theory of channel networks, based on the work of Hayes [4], as a means to derive necessary conditions for database representations of road networks. By exploring this example, we show that affordance-based logical definitions are a convenient method to capture essential properties of physical objects usually not present in their database representation, but appropriate to explain and define its structure.
\end{abstract}

Keywords: Ontology, Road network, Affordance-based theory, Naïve physics

\section{Physical Object Notions and Geographic Databases}

Affordance-based logical theories were inspired by Gibson's work [2] and have been used as a means for semantic analyses [7][8][12]. But can they also be used to classify representations of physical objects in a geographic database into affordance-based categories? For example, can an affordance-based specification of a certain kind of intersection be used to classify features in a road database? If the answer is positive, this provides an approach to semantically annotate the contents of databases and services.

We assume that a formal definition of a natural language term in a logical theory specifies a human category denoted by this term. Since each feature is an instance of a certain data type (a.k.a. feature type), we can focus on the evaluation of a data model with respect to category definitions. There are two major challenges in this approach: 
Limited Definability. Attempts to formally define terms of a natural or technical language face the "human knowledge soup" [11], so they suffer from overgeneralizations, abnormal conditions, unanticipated applications, and incomplete definitions. This usually means that (1) existing instances of a category are overlooked ("birds fly", but what about penguins?) and (2) non-instances are included ("birds fly", is then a bat also a bird?). Although this problem is generally unsolvable, it does not mean that incomplete definitions are useless. Ontologies are in fact meant to be partial specifications of conceptualizations [3]. Furthermore, as Hayes [5] has pointed out, a logical theory aiming to describe the physical world can be at most experimentally complete (see Israel [6]) or conceptually closed, so that everything that should be said can be said, and it is always doomed to include "ghostly" unintended models in its universe of meaning. One can handle this problem by tying the meaning of the theory's tokens to observational systems [5]. In our case we try to tie a physical theory to a data model, but this poses another severe problem.

Informational Incompleteness. Essential properties of the theory are not explicitly represented or excluded in the data model. Think about a computer application that has to figure out where to "go and get a coffee" by using a geometric data model of all objects in a room. One must deal with the difficulty of how to interpret a theory in an incomplete data model, because essential aspects of the task, like for example the information about the location of liquids in a room, are not present in it. This difficulty could be solved if the intelligent agent knew that liquids can be contained in a cup and if it could identify a cup. Therefore a solution to this problem can be appropriate knowledge representation [4]. For this we suggest the usage of an affordance-based theory, which allows to partially infer the missing types and their properties.

In this paper, we develop a conceptually closed theory of movements and supports in which it is possible to define the category road network by describing what actions its members must afford. From this definition it is then possible to infer and explain necessary structural properties of any road network data model. Our work presents a method for defining arbitrary categories of a road network database by explaining its structural properties using a specialized affordance theory. We intend to address more sophisticated examples like "road" and "junction" explicitly in the near future. As current data model standards for transportation networks, like ISO GDF, are rather informal descriptions, we furthermore see this work as a contribution to a formal domain ontology.

In the next section, we sketch our approach. In section 3, we introduce a common graph theoretic data model for navigational road network data. In section 4.1, we introduce the abstract concept of a history, which is the basic thing to exist in a physical world that has to cope with movements. A purposefully developed ontology of channels is introduced in section 4.2. In section 5, we introduce an affordancebased notion of a channel network and partially interpret the network theory in the graph data model. 


\section{Methodological Sketch}

Affordances say something about the kinds of physical actions that are possible in an environment. But affordances also say something about certain static aspects of the environment, which we call structure. Physical actions and structures are thought to be mutually dependent.

For our purpose, we need an affordance-based logical theory consisting of basic and derived types, each of which denotes a set of objects in the theory's universe, and some axioms and definitions that give meaning to them. We suppose there are two non-functional kinds of types structure and action. For our affordance-based theory to be useful, it should logically restrict the joint appearance of structures and actions in the conceptual affordance relation, such that if we restrict the structure part of the theory, the action part is restricted as a consequence, and vice versa. For example, if our physical universe of traffic had only rollerblades and skateboards as conveyances, then the whole accessible world's infrastructure would be restricted to $21 / 2$ dimensional solid objects with smooth surfaces.

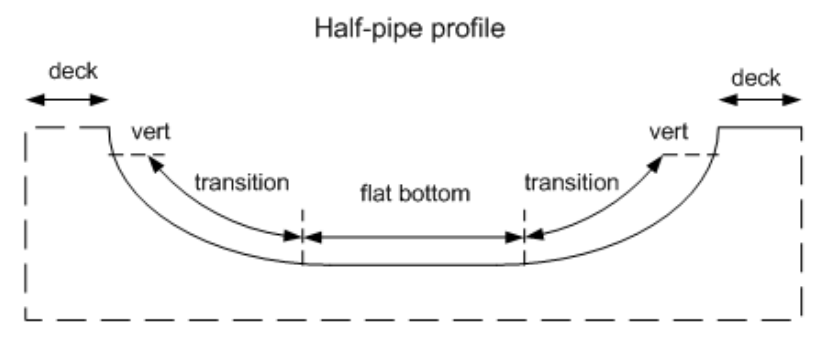

Fig. 1. A half-pipe in a skateboard world is an example of an affordance category.

Our task now is to define a category as a structure subtype in terms of the commonsense knowledge that comes with it, and then to interpret this definition in a data model. For example, in our skateboard world, we would like to define a "halfpipe" (compare FIGURE 1) in order to search for "half-pipe candidate" objects. We suppose that affordance-based theories are especially well suited for this purpose, because on the one hand, the type of afforded action is usually what we know a-priori about such a category, so it comes close to our commonsense knowledge about it. As a skateboarder, we perfectly know that a half-pipe is a thing that allows us to attain extreme speeds by an action called "pumping". On the other hand, we can assume that every member of such a category must afford this type of action, and therefore we have a convenient means of finding necessary conditions for an object to be in that category. So we can safely call every object that affords pumping actions a half-pipe. The most important aspect is nevertheless that the affordance-based theory allows us to infer what is usually not known a-priori for the category, which are the structural properties of the object that exist due to its afforded action. If we search for a halfpipe in our skateboard world, we may infer that everything which is similar to a 
certain upward concavity could be one. It is therefore possible to close the informational gap between incomplete knowledge about afforded actions and an incomplete data model of the affordance structure.

An affordance-based theory allows defining a physical object in affordance terms, that is defining its structural properties with the help of its afforded actions. These properties are expressed by the set of theorems about the defined structural subtype, which we call affordance theorems. A data model is said to partially satisfy the affordance definition, if there is a translation from every non-logical symbol of this model into the theory such that the translated tokens all satisfy the subset of affordance theorems that exist about them. We call this test weak data satisfiability of a data model.

The proposed method is considered to be useful in at least two respects (much in the spirit of analytic/deductive machine learning):

- In order to find reliable and operational (but necessarily incomplete) formal definitions of categories for databases

- In order to find domain theoretical explanations for structural properties of data base instances

In the remainder of the paper, we show that the data model introduced in the next section, which is a common representation of a road network (for details see [10]), satisfies the structural part of the affordance based definition of a road network developed in section 4 and 5 .

\section{A Common Road Network Data Model}

Let the road network $S=(N, L)$ be a directed graph with nodes $N$ and edges $L \subseteq N x$ $N$. For an edge $l=\left(n_{1}, n_{2}\right)$, let $l^{-}=n_{1}$ its incident source and $l^{+}=n_{2}$ its sink. A node stands for a street intersection and an edge for one direction of a street segment between two intersections. Note that a bidirectional street segment is represented by two parallel edges $l=\left(n_{1}, n_{2}\right)$ and $l^{\prime}=\left(n_{2}, n_{1}\right)$. A trace t consists of a sequence of edges $l_{1}, \ldots, l_{n}$ such that $l_{i}^{+}=l_{i+1}{ }^{-}$for all $i=1, \ldots, n-1$. Let $t^{-}=l_{1}^{-}$denote the source and $t^{+}=l_{n}^{+}$the sink of the trace $t$.

We call a road network embedded if we additionally have mappings point : $N \rightarrow P$ and line $: L \rightarrow 2^{P}$ where $P$ is the set of points in the Euclidian plane; the embedding is a simple linear curve in the Euclidian plane that geometrically connects the points of its two incident nodes. We assume that bidirectional street segments have the same geometry, i.e. line $\left(n_{1}, n_{2}\right)=\operatorname{line}\left(n_{2}, n_{1}\right)$.

Further, we refer to a subset $E \subseteq\left\{\left(l_{1}, l_{2}\right) \in L \times L \mid l_{1}{ }^{+}=l_{2}^{-}\right\}$as navigation relation for vehicle traffic (compare line graphs $L(S)$ [1] or linear dual graphs [14]): $\left(l_{1}, l_{2}\right) \in$ $E$ implies that it is possible (according to traffic rule and street construction) to drive from the segment $l_{1}$ into the segment $l_{2}$ crossing their incident node $n=l_{1}{ }^{+}=l_{2}^{-}$. A trace $t=l_{1}, \ldots, l_{n}$ of the road network $S=(N, L)$ is navigable iff $\left(l_{i}, l_{i+1}\right) \in E$ for all $i=$ $1, \ldots, n-1$. Note that navigable paths or traces are a true subset of all the paths in $S$, because there are certain junction types where not each possible path in $S$ is allowed (compare Scheider et al. [10] and FIGURE 2). 
We henceforth assume that $\mathrm{S}$ is connected by navigable traces, i.e. for all nodes $n$, $n^{\prime} \in N$, there exists a navigable trace $\mathrm{t}$ such that $t^{-}=n$ and $t^{+}=n^{\prime}$.

From this several properties follow. We call a network edge in $L$ graveyard if it has no outgoing navigation edge in the navigation relation $E$, that is $\left\{l \mid \neg \exists l_{2} \cdot\left(l, l_{2}\right) \in E\right\}$. We call a segment a factory if it is an element of the set $\left\{l \mid \neg \exists l_{1} \cdot\left(l_{1}, l\right) \in E\right\}$, Because $S$ is connected by navigable traces, we can exclude graveyards and factories in $S$, that is, each network edge in $S$ must both have at least one navigation tuple leaving the segment and one navigation tuple entering the segment. Furthermore, each node must have two appropriately directed adjacent segments in $S$ being a navigable tuple from $\mathrm{E}$, and hence the minimum degree of each vertex in $S$ is greater than or equal to 2 . So $\forall n \in N . \exists\left(l_{1}, l_{2}\right) \in E . l_{1}^{+}=l_{2}^{-}=n$.

$$
\begin{aligned}
& \boldsymbol{n}_{1}=\text { crossing intersection } \\
& \boldsymbol{n}_{2}=\text { diverging bifurcation: u-turn } \\
& \boldsymbol{n}_{3}=\text { diverging bifurcation: ramp } \\
& \boldsymbol{n}_{\mathbf{4 5}}=\text { diametrical bifurcation }
\end{aligned}
$$

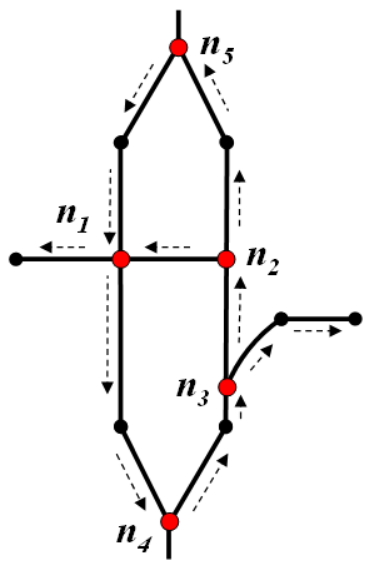

Fig. 2. Types of one-way intersection nodes for a dual carriageway road. Diametrical bifurcations $\left(\mathrm{n}_{4 / 5}\right)$ are examples of nodes with navigational restrictions.

For illustration purposes, let us consider some common road network features in this model. All non-parallel network edges in $S$ are called one-way edges. Navigation tuples connecting two bidirectional network edges are called $u$-turns. A set of two bidirectional network edges with one of its two vertices exclusively being part of a $u$ turn edge, is called dead-end. Vertices in $S$ can be e.g. diverging or converging bifurcations, diametrical bifurcations and crossings (compare FIGURE 2). Definitions of the higher level concepts bidirectional road, dual carriageway and junction (e.g. roundabout) are straightforward in this data model, compare [10].

\section{What Road Networks Afford}

The notions structure and action are domain dependent key concepts of every affordance definition, and therefore need a formal treatment consistent with our 
domain knowledge about the category. In the following theory, we elaborate such formalization for road networks in terms of the types flat support and supported movable history.

\subsection{Histories and Movements}

We base our theory on the logic of histories (for a detailed discussion we refer to [4]), and extend Hayes' theory to derive an ontology of movements, channels and networks.

Some remarks on the notational style. Sentences are written in predicate logic. For convenience, every expression is defined because invalid function applications have an undefined value and invalid predicate applications are defined to become false. Variables and constants are implicitly typed. Free variables are understood to be universally quantified. $\exists$ ! means the existence of exactly one instance. We always presuppose $\mathrm{a} \neq \mathrm{b}$ for two different symbols. Predicates are capitalized. Additionally, we use the ordinary operations on (point) sets and the usual Euclidian vector calculus. Some definitions and proofs are omitted and informally described in the text.

The principal argument of Hayes is, that in order to identify physical objects (and the things they are made of), we can cut space-time into histories. We describe histories as pieces of 4-dimensional space-time, $h \subset R^{4}$ (see appendix). We assume that histories and their lower dimensional projections in geographic space $G$ and time $T$ have well defined dimensionality, boundaries and interiors. A formal definition is given in the appendix. We can think of point histories as 0-dimensional, curve histories as 1-dimensional, surface histories as 2-dimensional and regular histories as 3-dimensional compact entities in geographical space that are "one-piece" and exist for an extended time interval. Note that the definition of the boundary and interior of a history $h$, denoted by $h$ and $h^{\circ}$, contrasts sharply with usual definition in point set topology.

We furthermore adopt the notion of a $\mathrm{face}^{2}$, and the function toso meaning that a piece-of-space is to the other side of a face of another piece-of-space (see appendix). We will use the binary predicate Disjoint to express that two pieces of space do not intersect, and Joined if they have just a face in common. We say that two pieces of space are InContact if they are Disjoint but there is only a free surface between the two (compare [4]).

A state is an instantaneous spatial slice of a history at a certain time-instant, that is the projection of a history $h$ into $G$ for a time instant $t$. A certain state is denoted by $h @ t$. As histories are topologically connected and bounded, there are two uniquely defined states called start and finish. Consequently, there are two unique time instants called begin and end (compare FIGURE 4).

Histories are not arbitrary subsets of Euclidian space; they are always thought to contain a physical object in each time instant. This object is different from the pieceof-stuff or the composite it is made of: we identify a river not by identifying the water

${ }^{2} \mathrm{~A}$ face of a piece-of-space $\mathrm{A}$ is a piece-of-space $\mathrm{F}$ of one lower dimension which forms part of A's boundary. 
in it, and we identify a car not by identifying its parts. We call a history solid, if its object is made of the same things in each moment in time, so that other histories cannot intersect with its interior. In order to specify this, we introduce the predicate Free and appropriately constrain the topological relations of solid (non-free) histories:

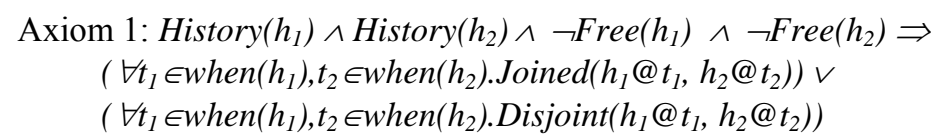

We furthermore assume that a free history does not include its faces, so it is equal to its interior (and therefore its faces can be either solid or free), whereas a solid history does (compare FIGURE 3).
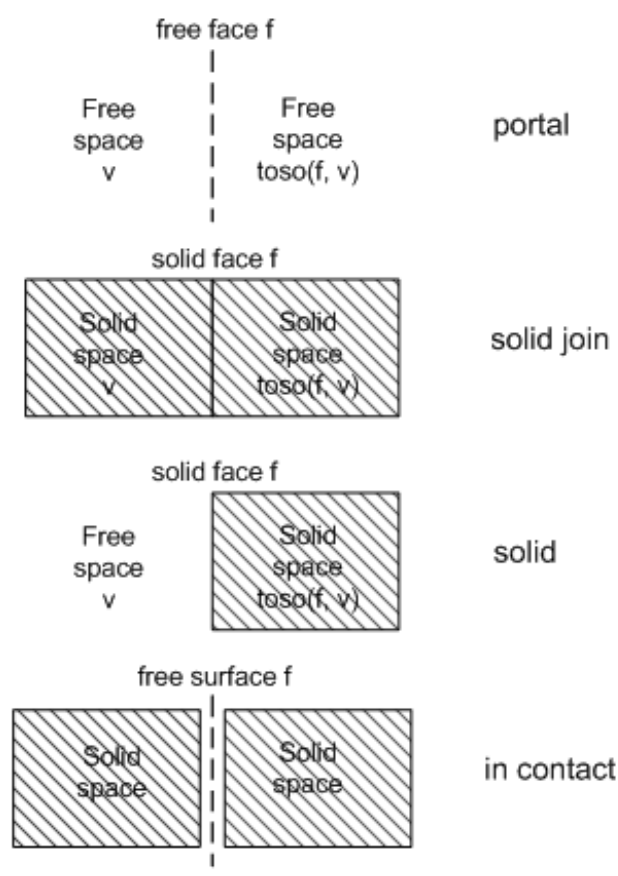

Fig. 3. Free and solid pieces-of-space and their faces, adapted from [4].

When $(h)$ denotes the time interval during which $\mathrm{h}$ takes place. Where $(h)$ denotes the spatial projection of $h$ in $G$ for all time instances, that is the place where the history "takes place". 
An episode $e$ of a history $h$, episode $(e, h)$, is defined as a subset of $h$ which has the same spatial extensionality as $h$ for a non-zero subinterval of when $(h)$ :

Def. 1: Episode(e,h) $\Leftrightarrow \operatorname{History}(e) \wedge$ when $(e) \subseteq w h e n(h) \wedge \forall t \in$ when(e).h@t=e@t
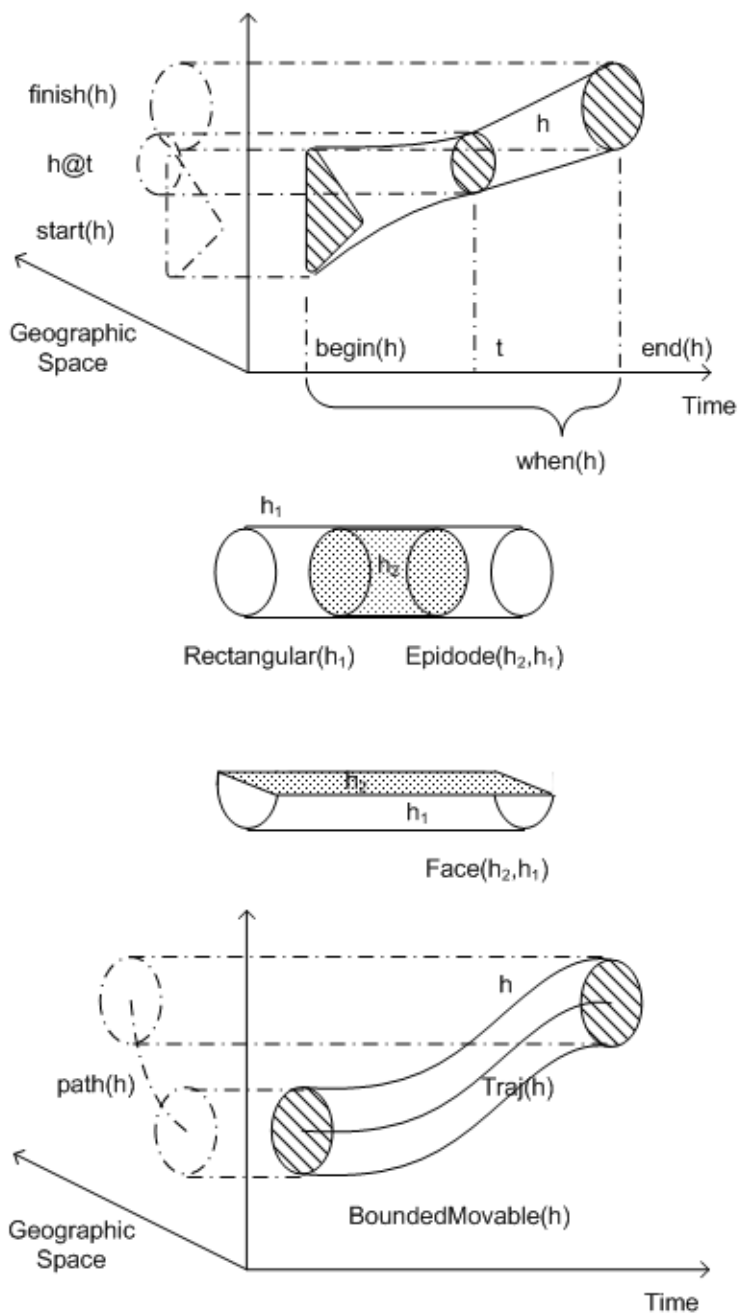

Fig. 4. Illustration of histories. Adapted from [4].

A history is said to be rectangular, if it doesn't change its geometric state in time:

Def. 2: Rectangular(h) $\Leftrightarrow \forall t_{1}, t_{2} \in$ when(h). $h @ t_{1}=h @ t_{2}$ 
A history is isometric if each pair of spatial points has a fixed Euclidian distance over all states. Let $f_{I}$ be a congruence isomorphism ${ }^{3}$ (isometric) function on Euclidian space $\mathrm{R}^{3}$ with distance function ${ }^{4} d$. A history is said to be isometric if it has a congruent shape for each pair of time instants:

Def. 3: Isometric $(h) \Leftrightarrow \forall t_{1}, t_{2} \in$ when(h). $\exists f_{I} . \forall s_{1} \in h @ t_{1} . \exists s_{2} \in h @ t_{2} . f_{I}\left(s_{1}\right)=s_{2}$

Clearly, rectangular(h) $\Rightarrow$ isometric(h). The function $\operatorname{Traj}_{x / t}{ }^{h}=\left\{<t_{i}, f_{I}^{t, t i}(x)>\mid t_{i} \in\right.$ when $(h)\}$ for some fixed $x \in h @ t$ and some fixed $t \in$ when $(h)$ denotes the trajectory of point $x$ at time $t$ of the isometric history $h$. Let point $x$ be the object's centroid. Clearly, as trajectories are point histories, they have episodes, start and finish, begin and end. Furthermore, we call the spatial projection of a trajectory, where(Traj), its path (compare FIGURE 4).

Now we can define the notion of differentiability for isometric histories. We call the first vector derivative of a trajectory the velocity vector of that time instant, $v_{f}(t)$. Differentiability is defined for all time points in the open time interval (denoted by ]...[) that the history encloses:

Def. 4: Differentiable $(h) \Leftrightarrow \operatorname{Isometric}(h) \wedge \forall t \in]$ begin( $h)$;end( $h)[$.

$$
\lim _{\Delta t \rightarrow 0}\left(\operatorname{Traj}^{h}(t+\Delta t)-\left(\operatorname{Traj}^{h}(t)+v_{\text {Traj }}(t) * \Delta t\right)\right) / \Delta t=0
$$

This has the consequence that the trajectory does not have any "sharp corners" with sudden jumps in direction. Also, from differentiability follows continuity for the open time interval of history $h$.

Movable histories obviously enclose solid objects, which is the reason for their differentiable behavior in time. In naïve physics, the histories of solid objects cannot finish at once [4], because they first have to lose their form. Following this thought, we assume that a moving object is an endurant. For the scope of this paper, we exclude non-isometric destruction and construction histories, and therefore require the existence of moving objects to last forever by AXIOM 2. Start(h) and finish(h) of a movable history then are also faces of other connected histories and the velocities at these states always exist and can be zero. Let us call differentiable non-free histories movable(h):

Def. 5: Movable $(h) \Leftrightarrow$ Differentiable $(h) \wedge \neg$ Free $(h)$

It follows a quite obvious theorem for movable subepisodes:

Theorem 1: Movable(g) $\wedge$ Episode $(h, g) \Rightarrow$ Movable(h)

We write $\mathrm{h} \otimes \mathrm{h}$ ' for the connection of two movable histories, with $h$ and $h$ ' being connectable episodes:

\footnotetext{
3 That is, a combination of a linear translation and a rotation preserving relative distances between points.

${ }^{4}$ Function $\mathrm{f}_{\mathrm{I}}$ is isometric iff $\forall \mathrm{x}, \mathrm{y} \in \mathrm{G}$. $\mathrm{d}\left(\mathrm{f}_{\mathrm{I}}(\mathrm{x}), \mathrm{f}_{\mathrm{I}}(\mathrm{y})\right)=\mathrm{d}(\mathrm{x}, \mathrm{y})$
} 
Def. 6: $h \otimes h^{\prime}:=h h^{\prime}$ iff h'@begin=h@end ^Movable(h) ^Movable(h')

We say that a movable history is larger than another movable history, $h<$ superh, iff there is a connection $h^{\prime \prime} \otimes(h \otimes h ')=$ superh. Now we state an axiom of infinite existence of movable objects:

Axiom 2: Movable(h) $\Rightarrow \exists h^{\prime}$. Movable( $\left.h^{\prime}\right) \wedge h<h^{\prime}$

Now we are able to define movements. A movement is a movable history without zero velocity, which means that it is continuously changing its location from the beginning to the end of its history:

Def. 7: Movement $(h) \Leftrightarrow \operatorname{Movable}(h) \wedge \neg \exists t \in]$ begin $(h) ;$ end $(h)\left[. v_{\text {Traj }}(t)=0\right.$

Note that the so called Brownian motion, as it is nowhere differentiable, and many other kinds of motions, e.g. simulated motions on a network [13], would not be considered a movement.

Using THEOREM 1 it follows that the connected histories of a larger movable history are also movable. Then it is clear that those episodes could be rectangular as well as movements. Now let $h$ be a movement history and $h$ ' be a connected rectangular history. It follows from differentiability of $h \otimes h$ ', that the velocities of $h$ must ultimately converge to zero. In general we can always distinguish between movable histories that converge to zero, and therefore must have a connected rectangular history, and histories which do not, and therefore must have a connected movement history. We call the first ones bounded movable histories and the second ones unbounded:

Def. 8: Unboundedmovable(h) $\Leftrightarrow$ Movable $(h) \wedge t \in]$ begin $(h)$; end $(h)[$

$$
\wedge \neg \lim _{t \rightarrow \text { end(h) }} v_{\text {Traj }}(t)=\mathbf{0} \wedge \neg \lim _{t \rightarrow \text { begin }(h)} v_{\text {Traj }}(t)=\mathbf{0}
$$

Clearly, unbounded movable histories imply connected movements. This follows from AXIOM 2, DEFINITION 8 and everything that was said above:

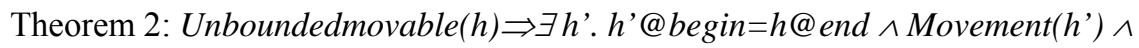
ᄏh'.h@begin=h'@end ^ Movement( $h$ '”)

We account for motions that can occur on a geographical scale, which we call friction dominated. These cannot be modeled in general. What can be modeled are aspects which exist due to the fact that the movement is constrained by its support infrastructure, which is the street surface.

\subsection{Supports and Channels}

Now we clarify the notion of a movement constraint. In doing so, we are mostly concerned with rectangular histories $h$, and for these, we write $h$ also as an abbreviation for where $(h)$. 
Friction dominated movements have supports. In terms of Newtonian physics, a support is a physical object whose spatial geometry is such that it provides a normal force for the contact force acting on the supported object. We define a support as a solid rectangular regular history with its 3-dimensional spatial extension in $G$ having a 2-D face (called a plateau) with a vertical component of its surface normal in every point. We therefore assume that the plateau of the support is a differentiable surface. Hayes' notion of a container is an example of a non-flat support [4]. If a flat support is projected into the geographic plane (denoted by the function plane), it is identical to the plane-projection of the plateau, so the plateau covers the cell.

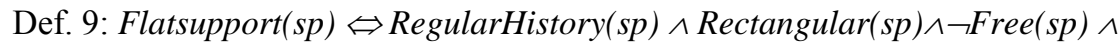
$\exists$ pl. plateau $(s p)=p l \wedge$ FaceOf $(p l, s p) \wedge$ DifferentiableSurface $(p l) \wedge$ $\forall s \in$ pl. $\exists v$.Verticalsurfacenormal $(p l, s)=v \wedge$

$\exists$ i. inside $(s p)=i \wedge$ RegularHistory $(i) \wedge$ Rectangular $(s p) \wedge$ Free $(i) \wedge$ FaceOf $(p l, i) \wedge$ plane $(p l)=\operatorname{plane}(s p)=\operatorname{plane}(i)$

For every support, we assume that there is a free space immediately above the plateau of a support, such that the plateau is a face of it and it is equal to the support's plane-projection. We call this space inside(sp). Unlike the inside of Hayes' open container, the height of inside(sp) is dependent on the height of the supported objects and is not determined by the support geometry.

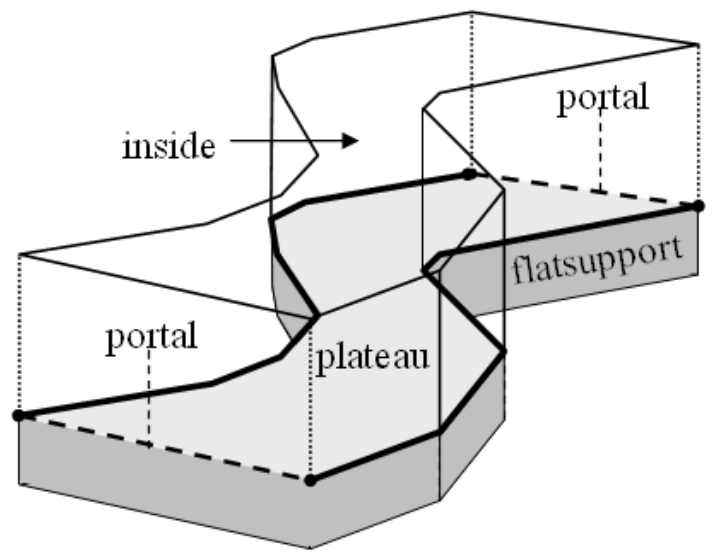

Fig. 5. An illustration of a flat support with two portals and two borders

For the definition of channels, we think of a portal as a free, differentiable face of the support's inside touching the plateau such that its plane-projection is a face of the plane-projection of the support (we say that the portal is on the boundary of the plateau): 
Def. 10: Portalof $(p, s p) \Leftrightarrow$ Free $(p) \wedge$ DifferentiableSurface $(p) \wedge$ Flatsupport $(s p)$

$\wedge$ FaceOf(p, inside(sp)) $\wedge$ FaceOf(plane $(p)$, plane $(s p))$

$\wedge \exists$ c. FaceOf(c, p) $\wedge$ FaceOf (c, plateau(sp))

In particular, we allow the formation of joined collections of free portals including their common free faces. These objects are again free surfaces. We abbreviate this operation by $\cup A$ for a set of histories $A=\{B, C, D\}$ :

Def. 11: Portal $(p) \Leftrightarrow \operatorname{Surface}(\iota p) \wedge$ Free $(\iota p) \wedge$ $\forall p_{i} \in p .(\exists s p$. Portalof(p,sp))

A movement is said to non-tangentially intersect a free differentiable surface in a point, iff its path intersects it in just that point, and the movement velocity vector does not converge to a vector lying in the tangential plane of the surface at that point:

Def. 12: Nti(h, $p, s) \Leftrightarrow$ Movable $(h) \wedge$ DifferentiableSurface $(p) \wedge$ Free $(p)$

$\wedge \exists s \in G$. $\left(\right.$ path $(h) \cap p=s \wedge \exists t, t_{i} \in$ when $(h) . \operatorname{Traj}^{h}(t)=s \wedge \lim _{t i \rightarrow t} v_{h}(t)=v_{h t}$ $\wedge\left(s+v_{h t}\right) \notin$ tangentialplane $\left.(p, s)\right)$

Note that from this definition it follows that the velocity vector cannot converge to zero (because then it would be in the tangential plane) and the point cannot lie at the non-differentiable boundary of the surface. A movement that non-tangentially intersects two surfaces in its start and finish therefore is unbounded. If a movement non-tangentially intersects the surface in an inner point of its trajectory, it is said to pass that surface:

Def. 13: Passing $(h, p) \Leftrightarrow \exists s \in G . N t i(h, p, s) \wedge s \notin \operatorname{start}\left(\operatorname{Traj}^{h}\right) \wedge s \notin \operatorname{finish}\left(\operatorname{Traj}^{h}\right)$

We state that a movement passing a portal has two connected episodes with one of them having a path being toso of the portal:

Theorem 3: Passing(h, p) ^P Portalof(p, sp) $\Rightarrow \exists e, e^{\prime} . e \otimes e^{\prime}=h \wedge$ $\operatorname{path}\left(e^{\prime}\right)^{\circ} \subseteq \operatorname{toso}(p$, inside(sp))

A proof of this theorem is not presented here, but because the curve does not intersect the surface in one of its end points, it can be divided into two connected parts. Then the only way that two parts of a differentiable curve can stay in the inside is to intersect it tangentially.

We furthermore restrict supported movement histories to always be in contact with a flat support, so we exclude flying movements, and having their path interior inside of the support's inside:

Def. 14: Supportedby $(h, s p) \Leftrightarrow$ Flatsupport $(s p) \wedge \operatorname{Movable}(h) \wedge$ $\forall t \in$ when(h). Incontact(h@t, where(sp)) $\wedge$ path(h) ${ }^{\circ} \subseteq$ inside(sp) 
We assume that a supported movable object has to stay supported for all its existence, so all its history connections have to stay in contact with a support.

\section{Def. 15: Supportedmovable $(h) \Leftrightarrow \exists s p$. Supportedby $(h, s p) \wedge$ $\forall h^{\prime} . h<h^{\prime} \Rightarrow$ Supportedmovable( $\left.h^{\prime}\right)$}

In particular, for a given supported movable history $\mathrm{h}$ and its support $\mathrm{sp}$, there always exists a unique largest supported history of $h$ with respect to $s p, h<$ largestsupported( $h, s p)$.

Now we can state that an unbounded supported movable history that nontangentially intersects a portal of its support implies the existence of a flat-joined support with a common portal. We call this theorem the theorem of unbounded movement support:

Theorem 4: Supportedmovable(h) ^ ᄏsp.Supportedby $(h, s p) \wedge$

$$
\begin{aligned}
& \exists p \text {.(Portalof }(p, s p) \wedge \exists s \in p \text {.Nti }(h, p, s) \\
& \Rightarrow \exists h^{\prime}, s p^{\prime}, p^{\prime} \text {. } \\
& \text { Movement(h') ^Supportedby }\left(h \otimes h^{\prime}, s p \cup s p \text { ')) } \wedge\right. \\
& \text { Portalof(p', sp') } \wedge s \in p^{\prime} \wedge \text { Passing }\left(h \otimes h^{\prime}, p\right)
\end{aligned}
$$

Proof: As the movement is supported, it is contained in a support's inside (by DEFINITIONS 14 and 15). Although it non-tangentially intersects a portal, it cannot pass that portal by THEOREM 3, because the portal is on the boundary, and the other side of inside(sp) must lie in its exterior. By DEFINITION 14, it therefore must intersect the portal in either its start or finish. Such a movement must be unbounded (see DEFINITION 13). As the movement is unbounded, by THEOREM 2, there must be a connected following (or preceding) movement. The connection of these movements must be passing the portal by DEFINITION 14, because the intersection point must be an inner point of the movement and it intersects the portal nontangentially. Then, by THEOREM 3, the connected movement episode is to the other side of the portal. By DEFINITION 15, the connections of the movement must be supported by a second support. As support histories cannot overlap (by AXIOM 1) and the movement is continuous (by DEFINITION 5), there must be a flat-joined history supporting the connected movement. And as the boundary of this support can only be passed at a portal, there must be a common portal that includes the point of non-tangential intersection. 


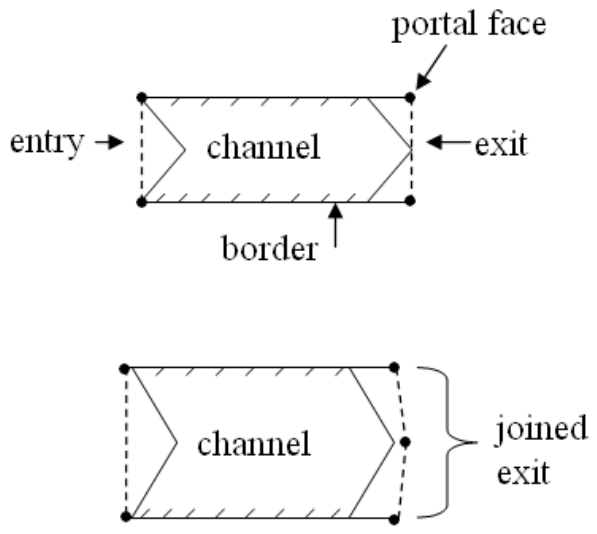

Fig. 6. Illustration (plane projection) of a channel. Exit and entry are portals, so they can consist of a chain of joined free differentiable surfaces.

Now we can think of a channel as a kind of flat support with entry and exit portals. We say that channels require their supported movements (or their larger movable histories) to actually leave at an exit portal and enter at an entry portal:

Def. 16: Channel(sp) $\Leftrightarrow$ Flatsupport(sp) ^

$\exists$ !entry, exit. Portal(entry) $\wedge$ Portal(exit) $\wedge$

$\forall p_{i} \in$ entry. $\forall p_{j} \in$ exit. Portalof $\left(p_{i}, s p\right) \wedge \operatorname{Portalof}\left(p_{i}, s p\right) \wedge$

$\left(\operatorname{Disjoint}\left(p_{i}, p_{j}\right) \vee \operatorname{Joined}\left(p_{i,} p_{j}\right)\right) \wedge$

$\forall$ h. (Movement $(h) \wedge$ Supportedby $(h, s p) \wedge m=$ largestsupported $(h, s p)$

$\Rightarrow\left((\right.$ Uexit $) \cap p a t h(m)=$ finish $\left(\operatorname{Traj}^{m}\right) \wedge \exists p \in$ exit.Nti $\left(m, p\right.$, finish $\left.\left.\left(\operatorname{Traj}^{m}\right)\right)\right) \wedge$

$\left((\right.$ Uentry $) \cap p a t h(m)=\operatorname{start}\left(\operatorname{Traj}^{m}\right) \wedge \exists p \in$ entry.Nti(m,p,start $\left(\right.$ Traj $\left.\left.\left.^{m}\right)\right)\right)$

\section{Channel Networks as Affordances}

Supported movable histories (DEFINITION 15) are the action type (compare section 2) of our affordance-based theory, whereas flat supports (DEFINITION 9) are the structure type. We derive necessary affordance conditions for a network by acting on the closed world assumption that all flat supports are part of one single network:

Axiom 3: $\exists$ ! Net. ChannelNetwork(Net) ^

$\forall$ SubNet. Flatsupport(SubNet) $\Rightarrow$ SubNet $\subseteq \cup$ Net

A channel network in terms of an action type can now be defined as a set of channels which must afford a movable history to connect every pair of points on their plateaus: 
Def. 17: ChannelNetwork(Net) $\Leftrightarrow \forall c \in$ Net.Channel(c)^ $\forall s_{1}, s_{2} \in \cup_{c \in N e t}$ Plateau(c). $\exists$ h. Supportedmovable(h) $\wedge$ plane $\left(\operatorname{start}(\operatorname{Traj})^{h}\right)=\operatorname{plane}\left(\mathrm{s}_{1}\right) \wedge$ plane $\left(\right.$ finish $\left.\left.(\operatorname{Traj})^{h}\right)\right)=\operatorname{plane}\left(\mathrm{s}_{2}\right)$

From DEFINITION 17 and the closed world assumption many plausible network theorems follow. In particular, it follows that every channel of a network must be connected to other channels at every one of its portals. Otherwise the network would exclude movements starting or ending at these portals because of DEFINITION 16 and THEOREM 4. This restricts the possibilities for channel configurations.

We illustrated possible flat-joined channel configurations in FIGURES 7, 8, 9, and one can see that well known road network feature types can be derived by flat-joining channels so as to form common portals. In this way, three-way intersections like bifurcations can be formed (FIGURE 8), as well as 4-way intersections (FIGURE 9).

(1) One way
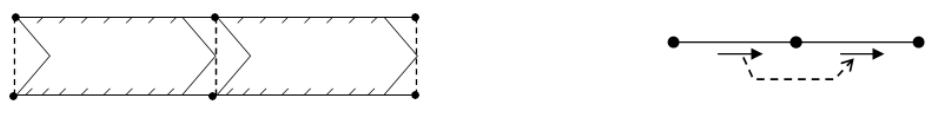

(2) Bidirectional way
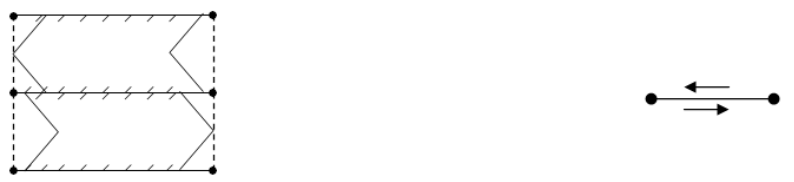

(3) Dead end
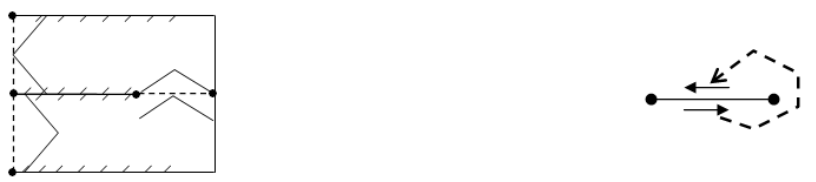

Fig. 7. Some important flat-joined configurations of channels (left) forming road features, and their translations in the graph data model (right) (dotted arrows mean navigation tuples).

It is astonishing that a certain type of configuration logically requires also the existence of so called periodic channels (see FIGURE 8). These are channels that must alternately cease to exist, because otherwise two logically necessary channels would intersect "at grade", which is prevented by AXIOM 1. In these cases we can presume the existence of a traffic regulator, like a traffic light.

Furthermore, we can obviously distinguish between channels that are connected to the same portal (see number 5 in FIGURE 8 and FIGURE 9), and ones that are connected to two different portals, which we will call inner and outer channels, 
respectively. We suspect also that it is provable in our theory that periodic channels are always inner channels.

On the right hand side of each figure, we included equivalent sub-graphs of the graph data model in section 3, translated into our theory by the following rules:

1. Let the set of network nodes $N$ in the graph $S$ be the set of portals of a network.

2. Let every directed edge of $S$ in $L$ be an outer channel of this network, such that the first vertex is the portal including the channel's entry and the second one is a different portal including the channel's exit. For two bidirectional edges in $S$, let their outer channels be joined at their border.

3. Let a navigation tuple in $\mathrm{E}$ mean that its two vertices, translated to outer channels, are either connected at a common portal or at a common inner channel.

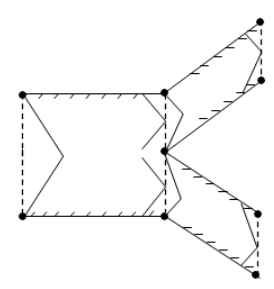

\section{(3) Diverging bifurcation}

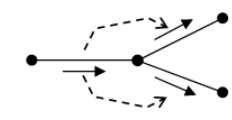

(4) Diametrical bifurcation
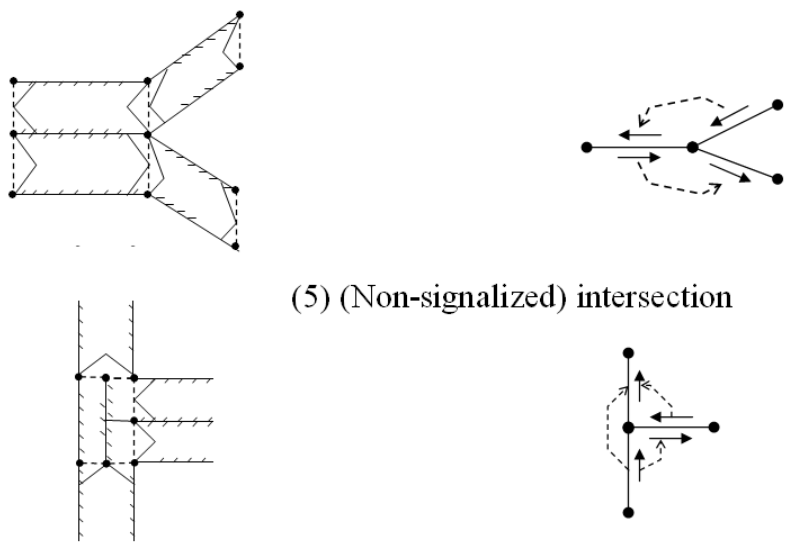

(5) (Non-signalized) intersection

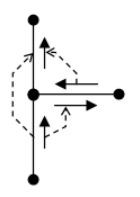

Fig. 8. Flat joined configurations of channels that form 3-valued vertices in the graph data model.

Let us denote the translations into our theory $\tau(\exp )$, with exp being a type (e.g. $N$ ) or a term (e.g. $n \in N$ ) of the graph data model. We now have an exact notion of the incompleteness of the data model: As portals are reduced to a vertex in the graph data model, the information about inner channels and periodic channels (the existence of 
traffic regulators) is lost. The portals of a channel also include its possible turn off directions, but these are modeled as edges of the navigation relation $E$.

If the translated data model of the set of edges $L, \tau(L)$, is a channel network, then the translation has to comply with the following affordance theorems:

Because of AXIOM 3 and DEFINITION 17, there cannot be other supports than channels of one single network. Clearly, as $\tau(S)$ consists of channels and portals of one network, there are no other supports existent in the data model.

(5) (Signalized) Crossing

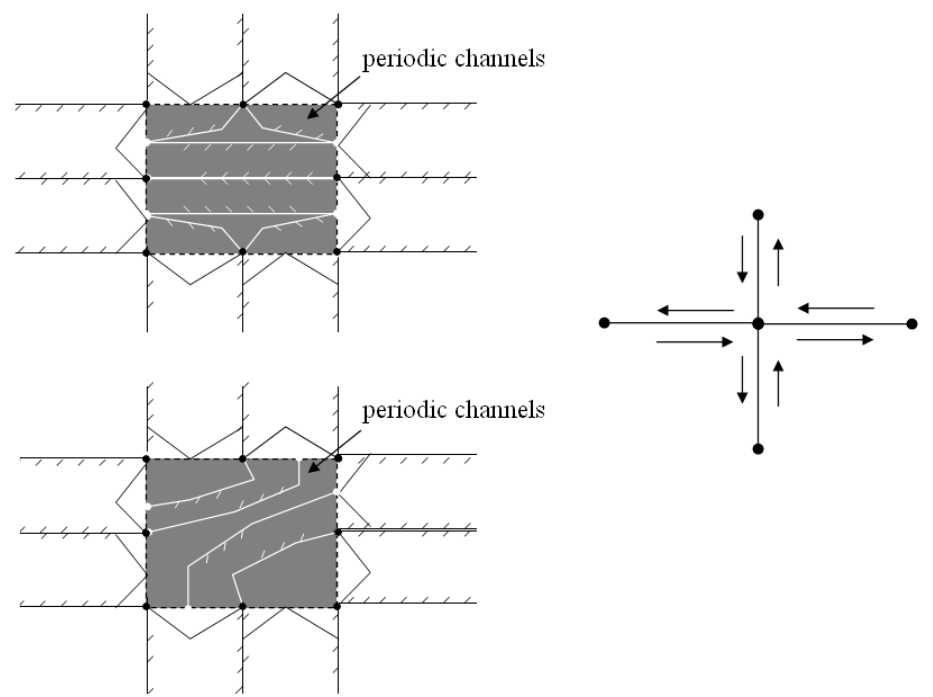

Fig. 9. Flat joined configurations of channels that form 4-valued vertices in the graph data model. These require the existence of periodic channels (signal period), because all channels meet at-grade.

Furthermore, every channel of the network must be reachable from every other channel by a chain of navigable connected channels. It follows from AXIOM 3 that a movement is supported by a chain of connected channels. According to DEFINITION 17 , there must exist a supported movement from every channel to every other one, and so there must also exist an appropriate chain of connected channels, q.e.d.. Now, is this theorem satisfied by the translated data model? As the edges of the graph $S$ are translated into outer channels, from the connectedness of $S$ by navigable paths, it follows that for each pair of outer channels, there must exist a chain of appropriately connected channels between them, because each tuple of $E$ is translated into either a pair of connected outer channels or a triple of connected inner and outer channels.

So, both theorems of the channel network are satisfied by the data model, and from these, all other mentioned characteristics of the data model follow, like the nonexistence of graveyards and factories and the minimum vertex degree of 2. 


\section{Conclusion}

Every attempt to classify representations of physical objects in a geographical database into categories is challenged by limited definability and informational incompleteness. We propose to use affordance-based logical theories in the sense of Naïve Physics in order to derive a set of necessary properties, called affordance theorems, for every data model that is supposed to represent such a category. On the one hand, the specification of the afforded action, in our example the movement connectivity of a channel network, comes close to the commonsense a-priori knowledge about the category. On the other hand, through the affordance relation, it becomes possible to derive and explain a-priori unknown structural properties of the physical object, in our example graph connectivity, minimal vertex degree and the non-existence of graveyards and factories.

The proposed method needs to be further elaborated: It is necessary to provide a means to make sure that the proposed set of affordance theorems for the partially interpreted types is in fact complete, so that it covers all derivable theorems about these types in the proposed theory. Furthermore, the more interesting categories are road and junction types, which could be defined by induced sub-graphs of the network.

\section{Appendix}

A subset of $\mathrm{R}^{\mathrm{m}}$ is bounded if it is contained in an $\mathrm{m}$-ball of finite radius. A subset of $\mathrm{R}^{\mathrm{m}}$ is connected, if it cannot be partitioned into two disjoint nonempty closed sets. A topological n-manifold (with boundary) (compare [9]) is a Hausdorff space in which every point has a neighborhood homeomorphic to an open subset of Euclidean halfspace:

$$
\mathbb{R}_{+}^{n}=\left\{\left(x_{1}, \ldots, x_{n}\right) \in \mathbb{R}^{n}: x_{n} \geq 0\right\} .
$$

Let $\mathrm{M}$ be a n-manifold with boundary. The interior of $M$, denoted $\mathrm{M}^{\circ}$, of dimensionality $\mathrm{n}$, is the set of points in $\mathrm{M}$ which have neighborhoods homeomorphic to an open subset of $\mathrm{R}^{\mathrm{n}}$. The $\mathrm{n}-1$ dimensional boundary of $M$, denoted $\partial \mathrm{M}$, is the complement of $\mathrm{M}^{\circ}$ in $\mathrm{M}$. The boundary points can be characterized as those points on the boundary hyperplane $\left(\mathrm{x}_{\mathrm{n}}=0\right)$ of $\mathrm{R}^{\mathrm{n}}+$ under some homeomorphism.

A history is a subset $h \subset R^{4}$ of 4-dimensional space-time, with the first three real valued dimensions denoting geographical space $G:=R^{3}$, and the fourth real valued dimension denoting time $T:=R$, having the following properties:

1. $\mathrm{h}$ is a topological $n$-manifold (with boundary), with $1 \leq \mathrm{n} \leq 4$.

2. the projection of $\mathrm{h}$ into $\mathrm{T}$ (denoted by when $(\mathrm{h})$ ) is a non-degenerate interval, so it is connected and neither the empty nor the singleton set. We say that $h$ is "temporally extended".

3. each projection of $\mathrm{h}$ into $\mathrm{G}$ for any moment $\mathrm{t} \in \mathrm{T}$ (denoted by $\mathrm{h} @ \mathrm{t}$ ) is a bounded and connected subset of $\mathrm{G}$, so a history is said to be "spatially bounded" and "one piece". 
Every closed m-1 manifold embedded in $R^{m}$ (e.g. a closed 2-sphere in $\mathrm{R}^{3}$ ) divides the space $\mathrm{R}^{\mathrm{m}}$ into two unique disjoint open subspaces, one of which is the bounded interior and the other one being the unbounded exterior. As this is always the case for the boundary of an m-manifold (e.g. a 3-ball), for every boundary part $f$ (called "face") of this m-manifold, the function $\operatorname{toso}(f, e)$ is a bijective mapping between the two subspaces.

\section{References}

1. Diestel, R.: Graph Theory ( $2^{\text {nd }}$ edition). Graduate Texts in Mathematics 173. Springer, New York (2000)

2. Gibson, J.J.: The Ecological Approach to Visual Perception. Houghton Mifflin, Boston (1979)

3. Guarino, N.: Formal Ontology and Information Systems. In: Guarino, N. (ed.) Formal Ontology in Information Systems. FOIS'98, pp. 3-15, Trento (1998)

4. Hayes, P.J.: Naïve Physics I: Ontology for Liquids. In: Hobbs, J.R., Moore, R.C. (eds.) Formal Theories of the Commonsense World, Ablex Series in Artificial Intelligence, Norwood (1988)

5. Hayes, P.J.: The Second Naive Physics Manifesto. In: Hobbs, J.R., Moore, R.C. (eds.) Formal Theories of the Commonsense World, Ablex Series in Artificial Intelligence, Norwood (1988)

6. Israel, D.: A Short Companion to the Naïve Physics Manifesto. In: Hobbs, J.R., Moore, R.C. (eds.) Formal Theories of the Commonsense World, Ablex Series in Artificial Intelligence, Norwood (1988)

7. Jordan T., Raubal M., Gartrell B., Egenhofer M.: An Affordance-Based Model of Place in GIS. In: Poiker T., Chrisman N. (eds.): 8th Int. Symposium on Spatial Data Handling, SDH'98 , pp. 98-109, Vancouver (1998),

8. Kuhn, W.: An Image-Schematic Account of Spatial Categories. In: Winter, S., Duckham, M., Kulik, L., Kuipers, B. (eds.): Spatial Information Theory. 8th International Conference, COSIT 2007. LNCS, vol. 4736, pp. 152--168. Springer, Heidelberg (2007)

9. Lee, J.M.: Introduction to Topological Manifolds. Graduate Texts in Mathematics 202. Springer, New York (2000)

10.Scheider, S., Schulz, D.: Specifying Essential Features of Street Networks. In: Winter, S., Duckham, M., Kulik, L., Kuipers, B. (eds.): Spatial Information Theory. 8th International Conference, COSIT 2007. LNCS, vol. 4736, pp. 169--185. Springer, Heidelberg (2007)

11.Sowa, J.: Knowledge Representation. Logical, Philosophical, and Computational Foundations. Brooks Cole Publishing (1999)

12.Raubal M., Worboys M.: A Formal Model of the Process of Wayfinding in Built Environments. In: Freksa C., and Mark D. (eds.): Spatial Information Theory. International Conference COSIT '99, LNCS, vol. 1661, p. 381-399, Stade (1999)

13.Van de Weghe, N., Cohn, A.G., Bogaert, P., De Maeyer, P.: Representation of Moving Objects along a Road Network. In: Proc. 12th Int. Conf. on Geoinformatics , pp 187-194, University of Gävle (2004)

14.Winter, S.: Route specifications with a linear dual graph. In: D. Richardson, (ed.): Advances in Spatial Data Handling: Proc. 10th Int. Symp. Spatial Data Handling (SDH 2002), pp. 329-338, Springer, Heidelberg (2002) 